\title{
Estudio comparativo de las mejoras en las habilidades cognitivas y emocionales entre un currículo por objetivos y un currículo con enfoque por competencias en alumnos de la Facultad de Medicina Veterinaria y Zootecnia, UPCH, Perú
}

\author{
Comparative study of the improvements in cognitive and emotional skills between \\ an outcome-based curriculum and a competency-based curriculum in students of \\ the Faculty of Veterinary Medicine and Zootechnics, UPCH, Peru
}

\author{
Ricardo Grandez R., ${ }^{1,}$, Silvana Cáceres L. ${ }^{2}$, Claudia Miguel de Priego G. ${ }^{1}$, Ricardo \\ Rojas M. ${ }^{1}$, Elizabeth Hinostroza M. ${ }^{1}$
}

\section{Resumen}

Se presenta un estudio comparativo de las mejoras en las habilidades cognitivas y emocionales entre un currículo diseñado por objetivos, con innovación de metodologías activas (2012-II), y un currículo con enfoque por competencias (2018-I), en búsqueda de desarrollar las competencias genéricas y específicas mediante la mejora de estas habilidades. La evaluación de las habilidades cognitivas y emocionales se hizo adaptando la Encuesta del Inventario de Cociente Emocional Bar-On (EQ-i) de Reuven. La encuesta fue realizada en dos ocasiones en cada semestre. Los resultados se compararon para determinar si hubo mejoras entre la primera y segunda encuesta en cada semestre y entre ambos currículos. Así mismo, se compararon los resultados de indicadores académicos. El número de alumnos matriculados fue de 30 (2012-II) y 27 (2018-I) y la tasa de encuestados superó el $70 \%$. El resultado referido a la mejora de las habilidades cognitivas, solución de problemas, mostró una mejora de casi el doble entre la primera y segunda encuesta en ambas propuestas curriculares. En habilidades emocionales, manejo de estrés, se observó un impacto negativo en el semestre 2012-II, mientras que en el semestre 2018-I hubo

${ }^{1}$ Sección de Biociencias y Ciencias Clínicas, Departamento Académico de Medicina Veterinaria y Zootecnia, Facultad de Medicina Veterinaria y Zootecnia, Universidad Peruana Cayetano Heredia, Lima, Perú

${ }^{2}$ Facultad de Psicología, Universidad Peruana Cayetano Heredia, Lima, Perú

${ }^{3}$ E-mail: rgrandez@hotmail.com

Recibido: 12 de febrero de 2019

Aceptado para publicación: 26 de septiembre de 2019 
una mejora, posiblemente debida al mayor acompañamiento al alumnado en las actividades realizadas en el currículo con enfoque por competencias. En logros académicos hubo una mejora en el promedio general entre los semestres y una disminución significativa de la desaprobación entre los semestres 2012-II y 2018-I. Se puede concluir que la implementación de un currículo con enfoque de competencias, con metodologías activas de enseñanza, permitió la mejora en las habilidades cognitivas y emocionales, de los logros académicos y una disminución significativa de la desaprobación en el curso.

Palabras clave: habilidades cognitivas; habilidades emocionales; currículo veterinaria; veterinaria; enfoque por competencias

\section{Abstract}

A comparative study of the improvements in cognitive and emotional skills is presented between an outcome-based curriculum, with innovation of active methodologies (2012-II), and a competency-based curriculum (2018-I), aiming to develop generic and specific competences by improving these skills. The evaluation of cognitive and emotional skills was done by adapting the Reuven's Bar-On Emotional Quotient Inventory Survey (EQ-i). The survey was conducted twice in each semester. The results were compared to determine if there were improvements between the first and second surveys in each semester and between both curricula. Also, the results of academic indicators were compared. The number of students enrolled was 30 (2012-II) and 27 (2018-I) and the respondent rate exceeded $70 \%$. The result referred to the improvement of cognitive skills, problem solving, showed an almost double improvement between the first and second surveys in both curricular proposals. In emotional skills, stress management, a negative impact was observed in the 2012-II semester, while in the 2018-I semester there was an improvement, possibly due to the greater support to the students in the activities carried out in the development, correction and feedback received in the competency-based curriculum. In academic achievements there was an improvement in the general grade average between the semesters and a significant decrease in the disapproval between the two semesters. It can be concluded that the implementation of a competence-based curriculum, with active teaching methodologies, allowed the improvement in cognitive and emotional skills, improvement of academic achievements and a significant decrease in the failure of the course.

Key words: cognitive skills; emotional skills; veterinary curriculum; competency-based approach

\section{Antecedentes}

La sumilla del curso de Medicina de Animales de Compañía (MAC) lo define como: "Curso de naturaleza teórico-práctico cuyo propósito es contribuir en la formación integral del Médico Veterinario mediante el estudio de las enfermedades de los diversos sistemas orgánicos de los animales de compañia, con énfasis en la etiología, las manifestaciones clínicas, la fisiopatología, los métodos diagnósticos $y$ los procedimientos terapéuticos a realizarse en la solución médica de estos». Este es un curso semestral obligatorio de la carrera de Medicina Veterinaria y Zootecnia de la Facultad de Medicina Veterinaria y Zootecnia (FAVEZ) de la Universidad Peruana Cayetano Heredia (UPCH), en Lima, Perú. 
El Articulo 40 de la Ley Universitaria indica que las carreras se pueden diseñar según módulos de competencia profesional, y que dichos módulos deben permitir la obtención de un certificado para facilitar su incorporación al mercado laboral; y que el currículo se debe actualizar cada tres años o cuando sea conveniente, según los avances científicos y tecnológicos; en tanto que el Artículo 39 de la ley indica que el régimen de estudios se establece preferentemente bajo el régimen semestral, por créditos y con currículo flexible; definiendo un crédito académico como equivalente a un mínimo de 16 horas lectivas o el doble de horas prácticas (Ley N. $\left.{ }^{\circ} 30220,2014\right)$. En virtud de ello, la FAVEZ implementó un nuevo plan curricular, modificando el currículo diseñado por objetivos (Plan Curricular 2007) que comprendía 2 horas de teoría, 2 horas de práctica por semana y 3.0 créditos académicos, por un currículo diseñado con enfoque por competencias (Plan Curricular 2014) que comprende 2 horas teóricas, 4 horas de práctica por semana y 4.0 créditos académicos.

El curso de MAC ha sufrido modificaciones paulatinas que lo han ido diferenciando de la enseñanza tradicional universitaria y, pese a su diseño por objetivos, fue guiado hacia un curso práctico-teórico clínico vivencial, con innovación de metodologías activas de enseñanza, tales como el aprendizaje basado en problemas (Dodd, 2007; Yoo y Park, 2014), resolución de casos clínicos (Andreu et al., 2004), desarrollo del pensamiento crítico y de la creatividad, revisión, interpretación y análisis de información científica (Kennedy, 2007); actividades contextualizadas en la práctica real y actividades de apoyo social (Folgueiras y Martínez, 2009), y tecnologías de transferencia de la información científica (Báez, et al., 2006). La implementación de estas estrategias pedagógicas permitió una más fácil adaptación al nuevo plan curricular con enfoque por competencias instaurado a partir del año 2014, e implementado por primera en el curso del semestre 2017-I.
En esta nueva propuesta se consideraron las siguientes competencias genéricas: a) demuestra comportamiento y compromiso ético, b) resuelve problemas con rigor científico, c) se desempeña eficientemente en contextos socioculturales y profesionales diversos, y d) demuestra compromiso y responsabilidad con su entorno social y medio ambiente. Así mismo, las competencias específicas son: a) integra los conocimientos adquiridos y los relaciona en la búsqueda de la solución médica de las diversas enfermedades que afectan a los animales de compañía, propiciando la capacidad analítica que permita el constante aprendizaje de los conocimientos para la solución de los problemas médicos necesarios para el buen desempeño profesional; b) propone y discute la etiología, manifestaciones clínicas, fisiopatología, métodos diagnósticos y alternativas terapéuticas de las diversas enfermedades que afectan a los animales de compañía; c) investiga, analiza y discute información científica y relevante dirigida a la solución de los diversos problemas médicos de los animales de compañía; d) adquiere capacidades, habilidades y destrezas en la ejecución de procedimientos clínicos, diagnósticos rutinarios e imagenológicos y procedimientos terapéuticos, mediante prácticas dirigidas y seguimientos de casos en la Clínica Veterinaria Docente Cayetano Heredia (CVDCH); y e) ejercita las actividades de investigación mediante el estudio de prevalencias, casos clínicos, estudios retrospectivos y otras modalidades a partir de los casos de CVDCH de la FAVEZ.

Se puede definir el currículo diseñado por objetivos o tradicional, como la acumulación de saberes conceptuales que plantea propósitos generales del proceso pedagógico y didáctico consensuados con la sociedad, estableciéndose más como una intención o propósito; en tanto que el currículo con enfoque por competencias constituye procesos generales contextualizados, referidos al desempeño de la persona, estableciéndose más como un producto que una intención (Tobón, 2006; MINEDUC, 2010; Sosa y Capote, 2014). 
Bar-On (1997), Cortés et al. (2002) y Extremera et al. (2004) definen la inteligencia emocional como un conjunto de habilidades personales, emocionales y sociales y de destrezas que influyen en nuestra habilidad para adaptarnos y enfrentar las demandas y presiones del medio. Como tal, la inteligencia no cognitiva es un factor importante en la determinación de la habilidad para tener éxito en la vida, influyendo directamente en el bienestar general y en la salud emocional. El Inventario de Cociente Emocional de Reuver Bar-On fue aplicado en los alumnos del curso de MAC en dos planes curriculares con el objetivo de detectar el nivel de desarrollo de sus habilidades cognitivas y emocionales logradas.

El documento presenta un estudio comparativo de las mejoras en las habilidades cognitivas y emocionales entre un currículo diseñado por objetivos, con innovación de metodologías activas, y un currículo con enfoque por competencias; en búsqueda de desarrollar las competencias genéricas y específicas del curso a través de la mejora de las habilidades cognitivas y emocionales de los estudiantes participantes del curso.

\section{Metodología}

Los contenidos del curso, en ambos currículos, están divididos en tres unidades didácticas: a) Medicina Interna I (6 semanas), que incluye medicina del oído y de los sistemas respiratorio y digestivo; b) Medicina Interna II (6 semanas), que incluye medicina de los sistemas reproductor, cardiovascular endocrino, urinario y dermatología; y c) Tópicos en Medicina (5 semanas), que incluye medicina del sistema nervioso, oncología, oftalmología y afecciones del sistema osteoarticular.

Las estrategias didácticas comprenden las siguientes metodologías: Clases teóricas de los temas consignados en el sílabo, con el fin de consolidar los conocimientos necesarios; Clases prácticas de procedimientos terapéuticos, diagnósticos clínicos rutinarios e imagenológicos con el fin de afianzar capacidades, habilidades y destrezas en la práctica clínica; Revisión de la información científica de tópicos asignados, a partir de la cual se elaboran informes que conforman las lecturas de temas para evaluaciones cortas (pasos); y Trabajo de investigación clínica a partir de la casuística de la CVDCH-FAVEZ, el cual es sustentado en el aula. Además, el nuevo currículo consideró el Proyecto Social Comunitario, relacionados a la intervención y rol del médico veterinario zootecnista en la mejora de la salud de la comunidad y bienestar de los animales de compañía en un contexto social.

\section{La evaluación comprende las siguientes actividades:}

- Exámenes teóricos, que abarcan principalmente temas de desarrollo basados en casos clínicos y complementados con preguntas objetivas (alternativas múltiples, verdadero-falso, relacionar o elaboración de tablas) de acuerdo con las características del tema;

- Exámenes prácticos, consistentes en la resolución de problemas o casos clínicos que permitan el reforzamiento en la realización de procedimientos terapéuticos, diagnósticos clínicos, imagenológicos o en la elaboración de artículos de información científica realizados previamente en las clases prácticas, con el fin de afianzar y reforzar capacidades, destrezas y habilidades en la ejecución de estos;

- Revisión de información científica de tópicos asignados, que comprende la elaboración de un informe/resumen de hasta tres páginas sobre temas consignados en el sílabo, el cual es expuesto con ayuda audiovisual por grupos designados al azar;

- Prácticas calificadas, consistente en la entrega de un informe de ejercicios o actividades encargadas al final de cada práctica, con el fin de reforzar las competencias ejercitadas, y entregadas en la siguiente clase práctica; 
- Pasos cortos, que se realizan uno por unidad en la clase práctica y comprenden las lecturas asignadas y la revisión de información científica de tópicos otorgados en las fechas consignadas en el sílabo;

- Caso clínico, el cual es realizado a partir de la casuística de la CVDCH, donde los grupos preparan un caso realizando el seguimiento y las pruebas necesarias durante las 12 primeras semanas de clases;

- Informe del caso clínico, el cual es el resultado final de esta investigación clínica, entregada en versión electrónica en formato de artículo científico, siguiendo las indicaciones para autor de la revista Salud y Tecnología Veterinaria de FAVEZ-UPCH, y que correspondió a la nota del examen práctico de la tercera unidad;

- Sustentación del caso clínico, entre las semanas 13 a 16 del semestre. Comprende la calidad expositiva, el conocimiento del tema y la capacidad de respuesta a las interrogantes. Además, los asistentes son evaluados mediante sus aportes, comentarios o preguntas adicionales que realizan durante la presentación del tema.

Otras actividades formativas no calificadas en el currículo diseñado por objetivos, y consideradas como una actividad evaluada en el currículo con enfoque por competencias, consistieron en la participación en actividades de extensión social, donde se realiza apoyo a la comunidad a través de campañas de servicio veterinario, la asistencia al servicio de atención clínica en la CVDCH-FAVEZ, de forma voluntaria y rotativa, a fin de desarrollar conocimiento y experiencia a partir de casos de la práctica real e interactuar con médicos veterinarios de la práctica clínica y con los propietarios de los pacientes. Estas actividades fueron evaluadas en el currículo con enfoque por competencias mediante las siguientes actividades:
- $\quad$ Proyecto social comunitario, el cual se realiza como parte de un programa de tenencia responsable de mascotas en una comunidad de bajos recursos. A partir de estas actividades se elaboró un informe, tipo infograma, que se sustenta en la parte final del curso.

- Desarrollo de formatos de casos clínicos, el cual consistió en la entrega de los formatos de cada uno de los casos seguidos en la CVDCH, y a partir de los cuales se selecciona el caso para sustentación y elaboración del artículo científico.

Es importante aclarar que en el currículo diseñado por objetivos, las actividades de trabajos encargados y resolución de prácticas calificadas se desarrollaban como trabajo independiente en casa, ya que las sesiones de dos horas prácticas solo permitían el desarrollo limitado de las mismas. En tanto que en el currículo enfocado por competencias, la mayor parte de estas actividades se realizaba durante las sesiones prácticas; así como la supervisión, corrección y retroalimentación de las mismas, al disponerse de mayor tiempo (sesiones de cuatro horas continuas de prácticas).

La evaluación de las habilidades cognitivas y emocionales de los alumnos del curso fue realizada por la Oficina de Tutoría Académica y Consejería Psicológica de la Dirección de la Escuela de la Carrera Profesional de Medicina Veterinaria y Zootecnia, adaptando la Encuesta del Inventario de Cociente Emocional de Reuver Bar-On (Ugarriza, 2001). El estudio consideró los siguientes componentes de la Inteligencia Emocional: la solución de problemas como habilidad cognitiva, y el manejo del estrés como habilidad emocional.

La encuesta fue realizada en dos ocasiones cada semestre: la primera en agosto de 2012 y la segunda en noviembre de 2012 como parte del Semestre 2012-II para el currí- 
Cuadro 1. Resultado absoluto y relativo de las clasificaciones de los indicadores específicos de las habilidades cognitivas (solución de problemas) y emocionales (manejo del estrés) evaluadas en el curso de Medicina de Animales de Compañía, en currículo por objetivos (Semestre 2012-II) y currículo con enfoque por competencias (Semestre 2018-I). Facultad de Medicina Veterinaria y Zootecnia, Universidad Peruana Cayetano Heredia, Lima

\begin{tabular}{|c|c|c|c|c|c|c|c|c|}
\hline \multirow[t]{2}{*}{ Habilidades } & \multicolumn{4}{|c|}{$\begin{array}{l}\text { Currículo diseñado por objetivos } \\
\text { 2012-II }\end{array}$} & \multicolumn{4}{|c|}{$\begin{array}{l}\text { Currículo con enfoque por } \\
\text { competencias } \\
2018-\mathrm{I}\end{array}$} \\
\hline & \multicolumn{2}{|c|}{ Inicial } & \multicolumn{2}{|c|}{ Final } & \multicolumn{2}{|c|}{ Inicial } & \multicolumn{2}{|c|}{ Final } \\
\hline $\begin{array}{l}\text { Solución de } \\
\text { problemas }\end{array}$ & $\mathrm{n}$ & $\%$ & $\mathrm{n}$ & $\%$ & $\mathrm{n}$ & $\%$ & $\mathrm{n}$ & $\%$ \\
\hline desarrolladas & 6 & 25.0 & 13 & 54.2 & 9 & 33.3 & 13 & 65.0 \\
\hline adecuadas & 6 & 25.0 & 6 & 25.0 & 10 & 37.0 & 3 & 15.0 \\
\hline por mejorar & 12 & 50.0 & 5 & 20.8 & 8 & 29.6 & 4 & 20.0 \\
\hline \multicolumn{9}{|l|}{ Manejo del estrés } \\
\hline desarrolladas & 7 & 29.2 & 3 & 12.5 & 7 & 25.9 & 7 & 35.0 \\
\hline adecuadas & 7 & 29.2 & 5 & 20.8 & 7 & 25.9 & 7 & 35.0 \\
\hline por mejorar & 10 & 41.7 & 16 & 66.7 & 13 & 48.1 & 6 & 30.0 \\
\hline Matriculados & 30 & & 30 & & 27 & & 27 & \\
\hline Encuestas & 24 & 100.0 & 24 & 100.0 & 27 & 100.0 & 20 & 100.0 \\
\hline Encuestados & & 80.0 & & 80.0 & & 100.0 & & 74.1 \\
\hline
\end{tabular}

culo diseñado por objetivos. Así mismo, la primera encuesta para el currículo con enfoque por competencias se realizó en marzo de 2018 y la segunda en junio de 2018, como parte del semestre 2018-I. Los resultados de ambos semestres se compararon para determinar si hubo mejoras de las habilidades cognitivas y emocionales entre la primera y segunda encuesta de cada semestre, y entre ambos currículos. Asimismo, se compararon los resultados de las notas promedio, generales y por unidades, porcentaje de desaprobación del curso, y los resultados de la Evaluación General de la Docencia de cada semestre, que fue realizada por la Dirección Universitaria de Gestión de la Docencia (DUGED), UPCH.
Las habilidades cognitivas y emocionales se clasificaron como: Desarrolladas: personas con más probabilidades de sentirse satisfechas y eficaces en su vida, y con más capacidad para dominar los hábitos mentales que favorezcan su propia productividad, estabilidad y salud mental; Adecuadas: personas que demuestran ciertas cualidades requeridas para llevar a cabo determinadas misiones, teniendo éxitos y fracasos, de manera equilibrada; y Por mejorar: personas que no pueden poner cierto orden en su vida emocional, librando batallas que sabotean su capacidad de concentrarse en sus actividades y pensar con claridad (Dawda y Hart, 2000; Ugarriza, 2001; Bar-On et al., 2003). 


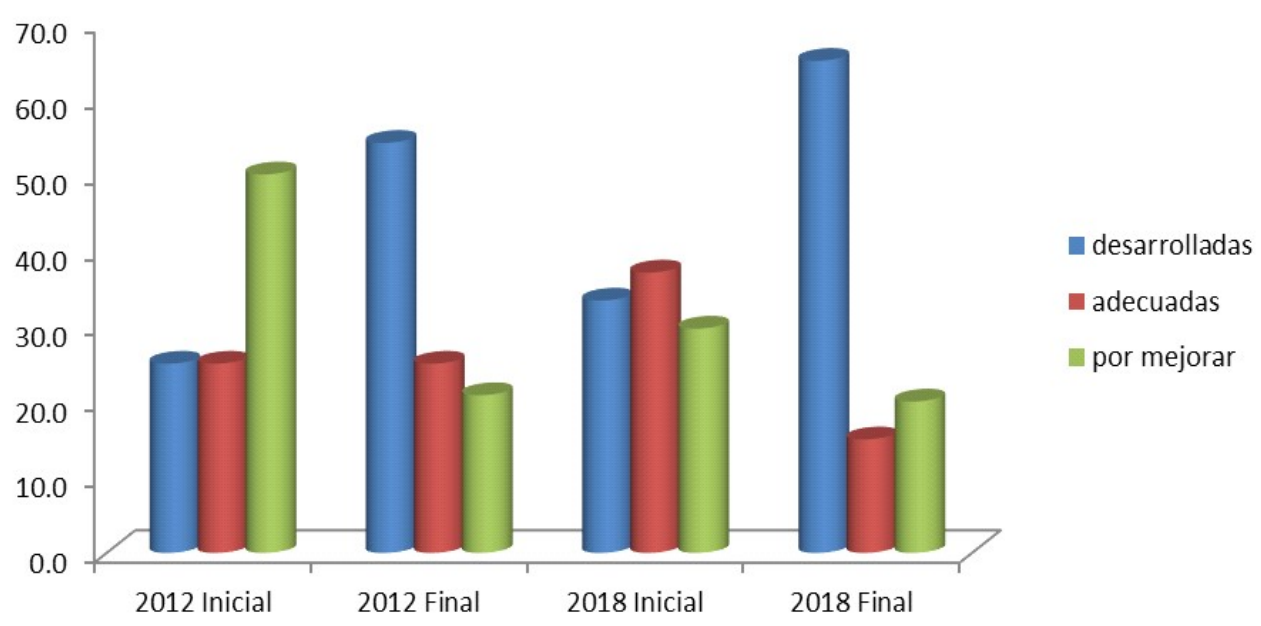

Figura 1. Clasificación de los resultados (Desarrollado, Adecuado y Por mejorar) de los indicadores específicos de las habilidades cognitivas (solución de problemas), evaluadas en el curso de Medicina de Animales de Compañía, en currículo por objetivos (Semestre 2012-II) y currículo con enfoque por competencias (Semestre 2018-I). Facultad de Medicina Veterinaria y Zootecnia, Universidad Peruana Cayetano Heredia, Lima. Resultados expresados en porcentaje

\section{Resultados y Discusión}

El número de alumnos matriculados fue de 30 y 27 en los semestres 2012-II y 2018-I, respectivamente; y el porcentaje de alumnos encuestados superó el $70 \%$ en todos los casos (Cuadro 1). El resultado referido a la mejora de las habilidades cognitivas y solución de problemas mostró una mejora de casi el doble en la clasificación desarrollada entre la primera y segunda encuesta en ambas propuestas curriculares; en tanto que la clasificación «Adecuada» se mantuvo entre la primera y segunda encuesta en el semestre 2012I, y la clasificación «Adecuada» $\mathrm{y}$ «Por mejorar» en ambos semestres disminuyó significativamente (Cuadro 1, Figura 1).

En cuanto a las habilidades emocionales, manejo de estrés, se observó un impacto negativo en el Semestre 2012-II, pues el número de alumnos con habilidades desarrolladas o adecuadas disminuyó considerablemen- te entre la primera y segunda encuesta, lo cual puede deberse a que, si bien las nuevas estrategias pedagógicas fueron efectivas, estas demandaron mucho esfuerzo por parte de los alumnos. Por el contrario, en el semestre 2018-I se observó una mayor clasificación de desarrolladas y adecuadas, en desmedro de la clasificación por mejorar (Cuadro 1, Figura 2); lo que pude ser explicado por el mayor acompañamiento en el desarro1lo, corrección y retroalimentación recibidas durante las secciones prácticas en el currículo con enfoque por competencias. Esto último permite al alumno superar su frustración y lograr satisfacciones en el desarrollo de estas actividades. $\mathrm{Al}$ respecto Sosa y Capote (2014) señalan que la evaluación debe ser vivida como parte del aprender y no como el mero reflejo de debilidades, buscando desarrollar una evaluación autentica: formativa, formadora y doblemente pedagógica; haciendo partícipe al estudiante y potenciando su autorregulación. 


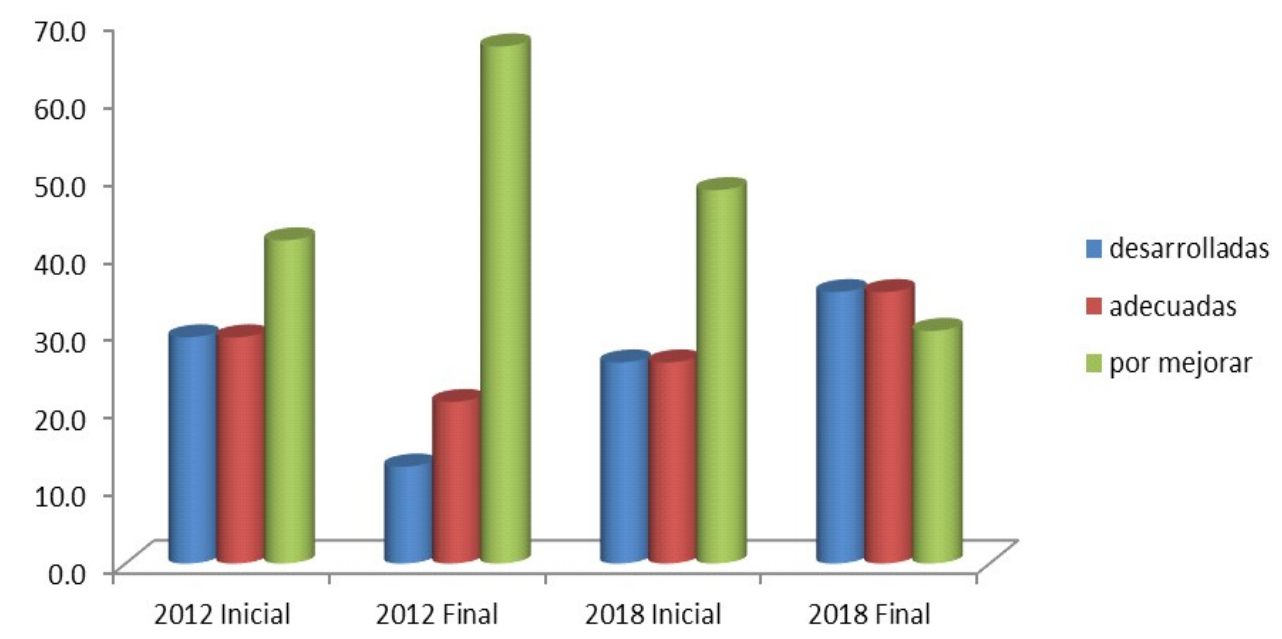

Figura 2. Clasificación de los resultados (Desarrollado, Adecuado y Por mejorar) de los indicadores específicos de las habilidades emocionales, el manejo del estrés, evaluadas en el curso de Medicina de Animales de Compañía en currículo por objetivos (Semestre 2012-II) y currículo con enfoque por competencias (Semestre 2018-I). Facultad de Medicina Veterinaria y Zootecnia, Universidad Peruana Cayetano Heredia, Lima. Resultados expresados en porcentaje

En cuanto a logros académicos, hubo una mejora en el promedio general entre los semestres 2012-II (11.7/20.0) y el 2018-I $(12.5 / 20.0)$. En forma similar, se observaron mejoras en las notas parciales de cada una de las tres unidades, mostrando una menor variación (rango entre el valor mínimo y el máximo) de notas en el general y en las unidades. Asimismo, se observó una disminución importante en la tasa de desaprobación del curso (de $23.3 \%$ a $7.4 \%$ en los semestres 2012-II y 2018- I, respectivamente). Por otro lado, la evaluación del curso por los alumnos mediante la encuesta de la Evaluación General de la Docencia presentó una leve disminución (de 3.6/4.0 a 3.5/4.0) entre los semestres 2012-II y 2018- I (Cuadro 2, Figura 3).

Los estudios de Pérez y Castejón (2006) indican la independencia que existe entre la inteligencia emocional y el cociente intelectual; no obstante, indican que existe correlación entre la inteligencia emocional y algunos indicadores de rendimiento académico. Por otro lado, Pendersen (2013) resalta la importancia del análisis de las emociones, la educación, y las relaciones humano-animales en la formación del Médico Veterinario, en tanto que Extremera y Fernández-Berrocal (2003) mencionan la necesidad de desarrollar programas de aprendizaje socioemocional basados en la capacidad de percibir, comprender y regular emociones. Es así que la innovación de las metodologías activas y técnicas educativas contextualizadas en la práctica real y actividades de apoyo social implantados en ambos currículos permitió el desarrollo de valores tales como el espíritu humanista, el altruismo, la responsabilidad social y el interés en el voluntariado en esta área de la carrera, con la consecuente mejora de sus capacidades emocionales y cognitivas.

Las metodologías activas de enseñanza adquieren importancia solo cuando el alumnado se desarrolla a partir de sus características personales y sociales. Para ello requieren de modalidades de enseñanza en que 
Cuadro 2. Resultado de las evaluaciones (notas de 0 a 20) y porcentaje de desaprobación y puntaje de la Encuesta Global de la Docencia (0-4) en el curso de Medicina de Animales de Compañía, en un currículo por objetivos (Semestre 2012-II) y currículo con enfoque por competencias (Semestre 2018-I). Facultad de Medicina Veterinaria y Zootecnia, Universidad Peruana Cayetano Heredia, Lima

\begin{tabular}{lcccccc}
\hline \multirow{2}{*}{$\begin{array}{l}\text { Resultado de } \\
\text { evaluaciones (0-20) }\end{array}$} & \multicolumn{3}{c}{$\begin{array}{c}\text { Currículo diseñado por } \\
\text { objetivos } \\
\text { 2012-II }\end{array}$} & \multicolumn{3}{c}{$\begin{array}{c}\text { Currículo con enfoque por } \\
\text { competencias } \\
\text { 2018-I }\end{array}$} \\
\cline { 2 - 7 } & Nota & Mínimo & Máximo & Nota & Mínimo & Máximo \\
\hline \multicolumn{1}{c}{ General } & 11.7 & 6.0 & 15.8 & 12.5 & 10.5 & 15.8 \\
Unidad 1 & 12.2 & 7.9 & 16.2 & 12.3 & 11.3 & 16.4 \\
Unidad 2 & 11.3 & 5.0 & 15.1 & 11.9 & 8.1 & 16.1 \\
Unidad 3 & 11.5 & 4.9 & 16.1 & 13.6 & 8.5 & 16.6 \\
Matriculados (n) & & 30 & & & 27 & \\
Aprobados (n) & & 23 & & & 25 & \\
Desaprobación (\%) & & 23.3 & & & 7.4 & \\
E. Global Docencia (0-4) & & 3.6 & & & 3.5 & \\
\hline
\end{tabular}

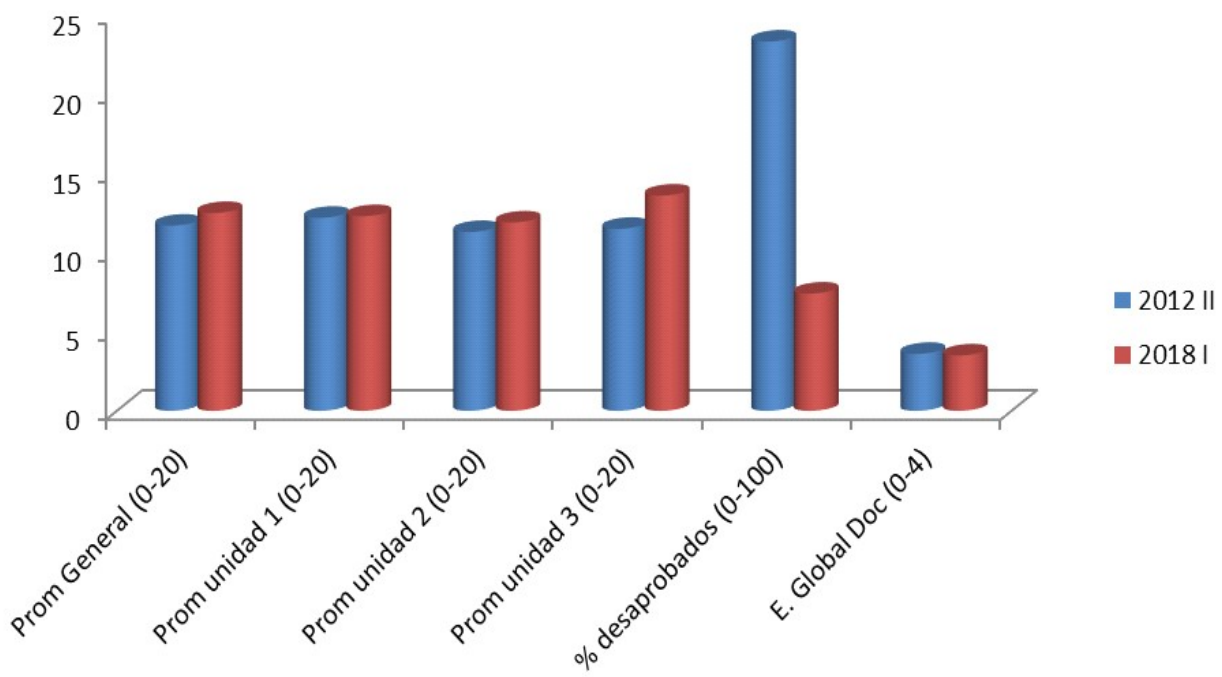

Figura 3. Resultado de las evaluaciones (notas de 0 a 20), porcentaje de desaprobación en el curso de Medicina de Animales de Compañía, y resultado de la encuesta de Evaluación Global de la Docencia (0-4.0) en un currículo por objetivos (Semestre 2012-II) y un currículo con enfoque por competencias (Semestre 2018-I). Facultad de Medicina Veterinaria y Zootecnia, Universidad Peruana Cayetano Heredia, Lima 
el alumno haga propios los objetivos en base a sus intereses personales, y que los aprendizajes de unos sean fuente de aprendizaje para otros e incluso que cada alumno se responsabilice de su aprendizaje y en cierta medida de sus compañeros (Salmerón, 2009). El currículo con enfoque por competencias permitió un mejor desarrollo de las metodologías educativas activas implementadas en el curso, ya que durante las prácticas y talleres, el papel del docente modificó su papel tradicional, de transmisor de contenidos, a un guía, moderador y dinamizador del aprendizaje de las competencias, como lo de describen Tobón et al. (2010); además de poder contextualizar las actividades de desarrollo de competencias en la comunidad, a través de actividades de apoyo social; y en el ejercicio de la carrera, mediante la asistencia al servicio de atención veterinaria, como lo describen Martínez y Folgueiras (2012).

Se dispone de escasa información respecto a programas de intervención centrados en la adquisición de habilidades y competencias de la inteligencia emocional a nivel de Latinoamérica (Pena y Repetto, 2008; Climént, 2014) y en el país (Ugarriza, 2001; Ugarriza y Pajares, 2008). Si bien el concepto de competencias es polisémico y complejo, ya que aún no existe una teoría unificada que sustente este enfoque y que se carece de experiencia en la puesta en práctica de este tipo de currículo (Moreno, 2012). Esta insuficiente experiencia hace que el currículo por competencias aun no constituya una alternativa válida para los modelos actuales. Se desarrollará un currículo por competencias cuando se aprenda a hacerlo, no cuando se enuncie en el discurso o en los papeles (Moreno, 2010). Del estudio se puede concluir que la implementación de un currículo con enfoque de competencias, con metodologías activas de enseñanza, permitió una mejora en las habilidades cognitivas y emocionales, mejora de los logros académicos y una disminución significativa de la desaprobación en el curso.

\section{Literatura Citada}

1. Andreu MA, Gonzales JA, Labrador MJ, Quintanilla I, Ruiz T. 2004. Método del caso, ficha descriptiva y necesidades. España: Univ. Politécnica de Valencia. $22 \mathrm{p}$.

2. Baéz EN, Mendoza D, Ramírez HC. 2006. Desarrollo de competencias TIC. México: Univ. Tecnológica de Nezahualcóyotl. $16 \mathrm{p}$.

3. Bar-On R. 1997. The emotional quotient inventory (EQ-i). Technical manual. Toronto, Canada: Multi-Health Systems.

4. Bar-On R, Tranel D, Denburg NL, Bechara A. 2003. Exploring the neurological substrate of emotional and social intelligence. Brain 126: 1790-1800. doi: 10.1093/brain/awg177

5. Climént J. 2014. Tipología de las competencias en educación veterinaria. Rev Inv Vet Perú 25: 293-316. doi: 10.15381/ rivep.v25i2.8503

6. Cortés JF, Barragán C, Vásquez ML. 2002. Perfil de inteligencia emocional: construcción, validez y confiabilidad. Salud Ment 25: 50-60.

7. Dawda D, Hart SD. 2000. Assessing emotional intelligence: reliability and validity of the Bar-On Emotional Quotient Inventory (EQ-i) in university students. Pers Indiv Differ 28: 797-812. doi: 10.1016/S0191-8869(99)00139-7

8. Dodd L. 2007. The impact of problembased learning on the information behavior and literacy of veterinary medicine students at University College Dublin. J Acad Libr 33: 206-216. doi: 10.1016/j.acalib.2006.12.008

9. Extremera N, Fernández-Berrocal P. 2003. La inteligencia emocional en el contexto educativo: hallazgos científicos de sus efectos en el aula. Rev Educ 332: 97-116.

10. Extremera N, Fernández-Berrocal P, Mestre JM, Guil R. 2004. Medidas de evaluación de inteligencia emocional. Rev Latinoam Psicol 36: 209-228. 
11. Folgueiras P, Martínez M. 2009. El desarrollo de las competencias en la universidad a través del aprendizaje y servicio solidario. RIED 2: 56-76.

12. Kennedy D. 2007. Redactar y utilizar resultados en el aprendizaje. Irlanda: University College Cork, Quality Promotion Unit. 103 p.

13. Martínez M, Folgueiras P. 2012. Competencias genéricas y específicas adquiridas por estudiantes de veterinaria en un proyecto de aprendizaje-servicio. En: II Jornada de Investigadores sobre Aprendizaje-Servicio. Buenos Aires, Argentina.

14. [MINEDUC] Ministerio de Educación. 2010. El currículo organizado en competencias: planificación de los aprendizajes. Ministerio de Educación de Guatemala, Dirección General de Currículo DIGECUR. $40 \mathrm{p}$.

15. Moreno T. 2010. El currículo por competencias en la universidad: más ruido que nueces. Rev Educ Superior 39: 77-90.

16. Moreno T. 2012. La evaluación de competencias en educación. Sinéctica 39. [Internet]. Disponible en: http:// www.sinectica. iteso.mx/index.php?cur $=39 \&$ art $=39 \_09$

17. Pena M, Repetto E. 2008. Estado de la investigación en España sobre inteligencia emocional en el ámbito educativo. Rev Electron Invet Educ 6: 401-420.

18. Pendersen H. 2013. Parasitic pedagogies and materialities of affect in veterinary education. Emotion Space Society 14: 50-56. doi: $10.1016 /$ j.emospa.2013.10.002
19. Pérez N, Castejón JL. 2006. Relaciones entre la inteligencia emocional y el cociente intelectual con el rendimiento académico en estudiantes universitarios. REME 22(9). [Internet]. Disponible en: http://reme.uji.es/articulos/numero22/ article6/numero\%2022\%-20article\%206\%20RELACIONS.pdf

20. Salmerón H. 2009. La formación por competencias en atención a la diversidad. Rev Educ Incl 2: 91-102.

21. Sosa A, Capote S. 2014. Modelo tradicional versus enfoque por competencias. [Internet]. Disponible en: https:// www.ces.edu.uy/index.php/anosabatico/143-modelo-tradicional-versusenfoque-por-competencias

22. Tobón S. 2006. Formación basada en competencias: pensamiento complejo, diseño curricular y didáctica. Ecoe. $266 \mathrm{p}$.

23. Tobón S, Pimienta JH, García JA. 2010. Secuencias didácticas, aprendizaje y evaluación de competencias. Practice Hall. 216 p.

24. Ugarriza N. 2001. La evaluación de la inteligencia emocional a través del Inventario de Cociente Emocional BarOn (I-CE) en una muestra de Lima Metropolitana. Persona 4: 129-160.

25. Ugarriza N, Pajares L. 2008. La evaluación de la inteligencia emocional a través del inventario de BarOn ICE: NA, en una muestra de niños y adolescentes. Persona 8: 11-58.

26. Yoo M-S, Park J-H. 2014. Effect of case-based learning on the development of graduate nurses' problem-solving ability. Nurse Educ Today 34: 47-51. doi: 10.1016/j.nedt.2013.02.014 